\title{
TUBERCULOSIS MAMARIA EN PACIENTE CON SIDA: A PROPÓSITO DE UN CASO.
}

\section{MAMMARY TUBERCULOSIS IN AIDS PATIENT: A CASE REPORT.}

\author{
Analía Silvana Chajud ${ }^{1,2,4}$, Adriana Almará ${ }^{1,3}$ Ana María Prestifilippo ${ }^{1,2}$.
}

\author{
1 Laboratorio de Microbiología. Hospital “San Martín”. Paraná. Entre Ríos. Argentina. \\ 2 Bioquímica \\ 3 Bioquímica especialista en Bacteriología Clínica \\ 4 Email de contacto: analiachajud@hotmail.com
}

\section{Conceptos claves}

Qué se sabe sobre el tema: La tuberculosis mamaria es una enfermedad rara que puede estar asociada al VIH/SIDA. La búsqueda de Mycobacterium tuberculosis como agente etiológico, es poco frecuente, ya que suele confundirse con otras infecciones oportunistas.

Qué aporta este trabajo: Este trabajo describe un caso clínico de tuberculosis mamaria de una paciente con VIH/SIDA, aportando con esto, un caso más de tuberculosis extrapulmonar, no dejar de sospechar la presencia de Mycobacterium tuberculosis, ya que aún sigue existiendo.

\section{Resumen:}

Introducción: la incidencia de tuberculosis mamaria en el mundo es muy baja. Esta patología puede ser primaria y más comúnmente secundaria. Se presenta como un foco extrapulmonar por invasión de Mycobacterium tuberculosis a la glándula mamaria. Método: presentamos el caso clínico de una mujer de 29 años con SIDA con tumoración de mama. Al momento de la consulta, también se auscultaron rales en ambos campos pulmonares. Se realizó radiografía de tórax, análisis de sangre, y tomografía computada de dicha tumoración de mama y axila. Al mes desde su ingreso al hospital, la tumoración fistulizó y drenó secreción purulenta de manera espontánea. Se tomó muestra y se envió para estudios anatomopatológico y microbiológico. También se realizó prueba de tuberculina (PPD). Resultados: la radiografía de tórax mostró un infiltrado intersticial difuso, sugestivo de neumonía por Pneumocystis Jirovecci. La tomografía computada de tumoración de mama y axila informó la presencia de múltiples adenopatías. El valor de PPD reportado fue de 5 milímetros, valor considerado como reacción positiva en pacientes con $\mathrm{VIH}$. El resultado de la anatomía patológica fue negativo para células neoplásicas Los resultados microbiológicos fueron: Mycobacterium tuberculosis, sensible a Isoniacida, Estreptomicina, Pirazinamida (PAS), Etambutol y Rifampicina. Conclusión principal: En base a los resultados del informe de laboratorio se realizó el diagnóstico de tuberculosis pulmonar, ganglionar y mamaria. La tuberculosis pulmonar en pacientes con SIDA, puede dar imágenes radiográficas no características, como en este caso que fue confundido en un primer momento con neumonía por Peumocystis Jirovecci.

Palabras clave: tuberculosis; SIDA; mama.

\begin{abstract}
:
Introduction: The incidence of breast tuberculosis in the world is very low. This pathology can be primary and, more commonly, secondary. It is presented as an extrapulmonary focus due to Mycobacterium tuberculosis invasion in the mammary gland. Method: we present the clinical case of a 29 -year-old in AIDS woman a breast tumor. At the time of the consultation, rales were also heard in both pulmonary fields. A chest $\mathrm{x}$-ray, blood analysis, and computed tomography of breast tumor and armpit, were performed. One month after admission to the hospital, the tumor fistulized and drained purulent secretion spontaneously. A sample was taken and sent to anatomopathological and microbiological analysis. A tuberculin skin test (PPD) was also performed. Results: the chest X-ray showed a diffuse interstitial infiltrate, suggestive of Pneumocystis Jirovecci pneumonia. Computed tomography of the breast and armpit tumors reported the presence of multiple adenopathies. The PPD value reported was 5 millimeters; this rate is considered as a positive reaction in HIV patients. The result of the pathological anatomy for neoplasic cells was negative. The microbiological results were: Mycobacterium tuberculosis, sensitive to Isoniacid, Streptomycin, Pyrazinamide, Ethambutol and Rifampicin. Main conclusion: Based on the results of the laboratory report, the diagnosis was pulmonary and mammary tuberculosis. The pulmonary tuberculosis in AIDS patients, can give non-characteristic radiographic images. This case proved how the diagnosis could be confused with pneumonia by Peumocystis jirovecci in a first approuch.
\end{abstract}

Keywords: tuberculosis; AIDS; breast. 


\section{Introducción}

De acuerdo a Finkestein Viegas ${ }^{1}$ el conocimiento de la tuberculosis mamaria data desde la antigüedad. Sin embargo, recién en el año 1829, Astley Cooper describió por primera vez esta localización tuberculosa con el nombre scrofulous swelling of the breast (tumefacción escrofulosa de la mama); asimismo, Virchow en 1836, describió esta patología con el mismo título. En 1860 Lancereaux efectúa el primer diagnóstico firme con estudio histológico. Más tarde, en 1868, Cuneo detectó bacilos de Koch en el pus de la mastitis tuberculosa, y recién en 1881, Dubar describió su anatomía patológica.

La tuberculosis mamaria es rara en la actualidad, $(0,2 \%)$ de acuerdo a Finkestein Viegas. Esta patología puede ser primaria y más comúnmente secundaria ${ }^{1}$.

Se presenta como un foco extrapulmonar por invasión de Mycobacterium tuberculosis a la glándula mamaria $^{2}$ La enfermedad se manifiesta como un tumor e inflamación, por lo general en los cuadrantes inferiores, es de crecimiento lento, con dolor de mediana intensidad; en algunas ocasiones puede haber un granuloma (8\%), que puede desbridarse de manera espontánea según su localización y expulsar una secreción blanca amarillenta ${ }^{3}$.

Cuando existe compromiso pulmonar en pacientes con VIH/SIDA, la radiografía de tórax frecuentemente muestra imágenes muy poco familiares, que no hacen pensar en tuberculosis y que se confunden fácilmente con otras infecciones oportunistas del SIDA ${ }^{3}$.

El Mycobacterium tuberculosis o bacilo de Koch, puede alcanzar la glándula mamaria por diversas vías: a) vía hematógena, que permite explicar la mastitis tuberculosa primaria; b) inoculación directa (rara); c) infección a partir de los conductos; d) diseminación a partir de estructuras vecinas; e) diseminación linfática a partir de los ganglios traqueobrónquicos (contaminación del grupo paratraqueal y luego de la mama); f) infección a partir de los ganglios cervicales o braquiales ${ }^{4}$.

Por otro lado, Berger y Mandelbaum consideran cinco formas de presentación de tuberculosis mamaria: a) mastitis miliar aguda, secundaria a una TBC miliar generalizada y que se caracteriza por la diseminación de focos miliares en el tejido interlobular (intersticial), b) mastitis tuberculosa nodular; forma más común de tuberculosis mamaria donde se forma una lesión caseosa que se fija a las estructuras vecinas y luego produce fístula, c) mastitis tuberculosa nodular diseminada; tubérculos múltiples que se caseifican y produce fístula, d) mastitis tuberculosa esclerosa; más frecuente en las mujeres de edad de lenta evolución, diagnóstico diferencial con el carcinoma; e) mastitis obliterans; proliferación epitelial de los conductos seguida de necrosis y fibrosis periductal ${ }^{5,6}$. La fístula puede ser única o múltiple, y si asienta cerca del pezón, puede retraerlo. La adenopatía axilar está presente en la mitad de los casos ${ }^{7}$.

La mastitis tuberculosa puede ser la primera manifestación del SIDA. En este caso es mandatorio demostrar el bacilo ácido alcohol resistente mediante coloración de Ziehl-Neelsen y cultivo ${ }^{8,} 9$.

\section{Objetivos}

Presentar un caso clínico de una mujer con VIH/SIDA con una tumoración de mama y describir cómo se llegó al diagnóstico de tuberculosis mamaria.

\section{Materiales Y Métodos}

\section{Descripción de un caso clínico y métodos utilizados en su estudio y diagnóstico}

Una mujer de 29 años de edad con VIH/SIDA ingresó a la guardia de nuestro nosocomio para estudio de tumoración en mama derecha. Además presentaba: muguet oral, diarreas crónicas, distensión abdominal, tumoración en axila y mama derecha. En el cuadrante externo periaerolar de dicha mama se palpó un tumor de consistencia firme, móvil, no adherido, con planos profundos de bordes irregulares de $5 \times 7$ centímetros.

Se auscultaron rales en ambos campos pulmonares y se realizó una placa radiográfica de tórax. Debido a que los resultados de la placa fueron sugestivos de neumonía por Pneumocystis jirovecii, se inició tratamiento en forma empírica con Trimetoprima-sulfametoxazol. Cabe aclarar, que la paciente no presentaba antecedentes de tuberculosis pulmonar. 
Se le realizaron las siguientes determinaciones: hematocrito, hemoglobina, glóbulos rojos, glóbulos blancos y serologías para hepatitis B, C, Chagas y VDRL.

Una vez obtenidos los resultados de los análisis de sangre, se decidió la internación de la paciente. A los 7 días de la misma su estado era reservado, debido a varias complicaciones provocadas por el mismo SIDA y, por lo que se la trasladó a Unidad de terapia Intensiva.

Luego de 10 días la paciente pasó a sala común y a los 20 días desde su internación se le realizó una tomografía computada de tumoración de mama y axila.

Luego de permanecer 48 horas sin fiebre, y haber transcurrido un mes desde su ingreso al hospital, la tumoración fistulizó y drenó secreción purulenta de manera espontánea y repentina por lo que se realizó toma de muestra por punción aspiración desde piel sana. La muestra se envió para estudio anatomopatológico y microbiológico. A los 20 días se recuperaron bacilos ácido- alcohol-resistente del cultivo en medio Owaga. El aislamiento fue enviado para identificación y pruebas de sensibilidad al Laboratorio de Tuberculosis del Instituto Nacional de Enfermedades Respiratorias "Dr. Emilio Coni".

Se realizó prueba de tuberculina (PPD).

\section{Resultados}

El resultado de la placa radiográfica realizada fue sugestivo de neumonía por Pneumocystis jirovecii observándose un infiltrado intersticial difuso. Teniendo en cuenta la guía de Organización Panamericana de la Salud (OPS $)^{10}$ conforme la inmunosupresión aumenta, la presentación clínica se vuelve altamente atípica e inespecífica.

Ver cuadro № 1:

Tabla 1: Resultados de laboratorio

\begin{tabular}{cll}
\hline \multicolumn{1}{c}{ Laboratorio } & & \multicolumn{1}{c}{ Resultado } \\
\hline & Hematocrito & $20,50 \%$ \\
& Hemoglobina & $7,10 \%$ \\
Análisis hematológico & Gobulos rojos & $2.730 .000 / \mathrm{mm}^{3}$ \\
& Globulos blancos & $13.500 / \mathrm{mm}^{3}$ \\
& Neutrófilos & $90 \%$ \\
\hline Serología & Hepatitis B & Negativo \\
& Hepatitis C & Negativo \\
& Chagas & Negativo \\
\hline Hemocultivo & VDRL & Negativo \\
\hline Urocultivo & & Negativo \\
\hline Citopatología de células & & Negativo \\
neoplásicas & & Negativo \\
\hline Presencia de hongos y bacterias & & Negativo \\
comunes & & Positiva XXX \\
\hline Baciloscopía & &
\end{tabular}

El resultado de la tomografía computada de tumoración de mama y axila informó la presencia de múltiples adenopatías y algunas de las mismas, con centro necrótico quístico.

El valor de PPD reportado fue de 5 milímetros, valor que está dentro del rango considerado como reacción positiva en pacientes infectadas con VIH acorde a las normas técnicas 2013 del programa Nacional de Control de la Tuberculosis ${ }^{11,12,13,14 .}$

Las pruebas de sensibilidad permitieron identificar la cepa como Mycobacterium tuberculosis, sensible a Isoniacida, Estreptomicina, Pirazinamida (PAS), Etambutol y Rifampicina. 


\section{Discusión}

En pacientes con VIH la presentación de la tuberculosis puede ser inespecífica y atípica, sobre todo en las formas extrapulmonares. La tuberculosis pulmonar puede dar imágenes radiográficas no características, como en este caso que fue confundido en un primer momento con neumonía por Peumocystis jirovecii La tuberculosis mamaria, ya sea primaria o secundaria, es una enfermedad poco frecuente y de diagnóstico dificultoso.

En la actualidad, se considera prácticamente imposible detectar un caso de esta patología si no hay un estado de inmunocompromiso, como el presentado en este trabajo, donde el bacilo fue invadiendo los tejidos hasta llegar a la mama.

Es importante recordar que la tuberculosis aún está presente, y no dejar de sospecharla más aún en pacientes con VIH/ SIDA.

\section{Conclusión}

En base a los resultados del informe de laboratorio se realizó el diagnóstico de tuberculosis pulmonar, ganglionar y mamaria. Con ello, se indicó el inicio de tratamiento con triple asociación (Isoniazida, Rifampicina y Pirazinamida) más Etambutol y se realizó drenaje quirúrgico del absceso.

\section{Agradecimientos}

Quiero agradecer a mi instructora la Bioquímica Especialista en Bacteriología clínica Adriana Almará por el apoyo en la realización de este trabajo.

\section{Bibliografía}

1. Finkenstein Viegas ML, Lage S, Orsi HR, Castaño R, Maletti G, Gross C. Tuberculosis mamaria. Revista Argentina de Mastología 2011; 30(107): 141-147

2. Soto C, Vizcaíno I, Isarria S, Pastor MR. Tuberculosis mamaria. Descripción de los hallazgos de imagen en dos pacientes. Radiología. 2008; 50:518-21

3. Hernández G.A. Avances en Mastología. Editorial Universitaria. Enfermedades Inflamatorias de la Mama. 1 ed. Chile, 1992.131-36

4. Pabst Y. Enfermedades Inflamatorias de la Mama. Bd. Hosp. San Juan de Dios. Chile, 1992; 39(6):328-36

5. Fernández Cid A y colab. Patología mamaria. Procesos Inflamatorios de las glándulas mamarias. Salvat ediciones. Barcelona. España, 1982. 202

6. Zandrino F, Monetti F, Gandolfo N. Primary tuberculosis of the breast: a case report. Acta Radiol 2000; 41(1): 61 63.

7. Kakkar S, Kapila K, et al. Tuberculosis of the breast. A cytomorphologic study. Acta Cytol 2000; 44: 292-6.

8. Meral Sen, Canan Gorpelioglu, Mikdat Bozer. Isolated primary breast tuberculosis - report of three cases and review of the literature. Clinics, vol.64 no.6 São Paulo, June 2009.

9. American Thoracic Society and CDC. Diagnostic standards and classification of tuberculosis in adults and children. (PDF) Am J Respir Crit Care Med 2000; 161. http://ajrccm.atsjournals.org/ content/161/4/1376.ful

10. Organización Panamericana de la Salud. Coinfección TB/VIH: Guía Clínica. Versión actualizada - 2010 Washington, D. C.: OPS, (C) 2010.

11. Zerbini, E Programa Nacional de Control de la Tuberculosis: Normas Técnicas 2013 (et. al.); 4ta ed. - Santa Fe: Instituto Nacional de Enfermedades Respiratorias Dr. Emilio Coni.

12. CDC. Guidelines for preventing the transmission of Mycobacterium tuberculosis in health-care settings, 2005. MMWR 2005; 54 (No. RR-17).

13. CDC. Mantoux Tuberculin Skin Test: Training Materials Kit (2003).

14. CDC. Targeted tuberculin testing and treatment of latent tuberculosis infection. MMWR 2000; 49 (No. RR-6). http://www.cdc.gov/MMWR/PDF/rr/ rr4906.pdf 